\title{
Chronic Kidney Disease in Older People: Physiology, Pathology or Both?
}

\author{
Ahmed H. Abdelhafiz ${ }^{\mathrm{a}}$ Siobhan H.M. Brown ${ }^{\mathrm{a}} \quad$ Aminu Bello $^{\mathrm{b}}$ Meguid El Nahas ${ }^{\mathrm{b}}$ \\ a Department of Elderly Medicine, Rotherham General Hospital, Rotherham, and bSheffield Kidney Institute, \\ Sheffield, UK
}

\section{Key Words}

Age $\cdot$ Chronic kidney disease $\cdot$ Cardiovascular disease

\begin{abstract}
The global population is aging due to a reduction in youthful deaths and an extension of the later stages of life. With aging comes a decline in the physiologic functions of various organs and systems. Vascular aging is associated with structural and functional changes of the arterial wall leading to loss of elasticity and compliance. Renal vasculature is not spared as aging is associated with arterial, arteriolar and capillary, glomerular changes (glomerulosclerosis). It is likely that age-related vascular changes are linked to the decline in renal function observed with aging. These changes occur at varying stages of aging depending on predisposing genetic factors and associated life course exposure to cardiovascular risk factors including hypertension and diabetes. The decline in renal function with 'normal' aging in the absence of associated progressive cardiovascular disease is slow and does not seem to be of major clinical significance. The current definition of chronic kidney disease (CKD), including microalbuminuria, and the method of estimation of glomerular filtration rate have inadvertently resulted in an exaggerated prevalence of CKD in the elderly. This is combined with the fact that most of the studies showing decline
\end{abstract}

in renal function with aging are limited by the absence of a correction for associated comorbid confounding factors, resulting in difficulty separating the effect of physiological aging on kidney function from pathological aging due to comorbidities. Such a correction is difficult, if not impossible, to objectively construct. We suggest that only those fractions of older patients with underlying progressive vascular pathology likely to involve the kidneys will, in the future, warrant attention to reduce vascular risk and the associated kidney damage.

Copyright $\odot 2010$ S. Karger AG, Basel

\section{Introduction}

The average age of the global population is steadily increasing and the number of people above the age of 80 years is growing faster than any other age group [1]. Chronological age may be different from biological age. Certain biological variables like graying of hair, skin elasticity and arterial function (vascular aging) correlate in a linear fashion with chronological age [2], whereas other biological markers such as lung capacity, hearing and cognitive function as well as visual acuity do not necessarily equate with chronological age. Advancing age is recognized as a major non-modifiable risk factor for car-

\section{KARGER}

Fax +4161306 1234 E-Mail karger@karger.ch www.karger.com
Dr. Ahmed H. Abdelhafiz

Department of Elderly Medicine

Rotherham General Hospital, Moorgate Road

Rotherham S60 2UD (UK)

Tel. +44 1709307 576, Fax +44 1709304 419, E-Mail ahmedhafiz@hotmail.com 
diovascular disease (CVD) [3]. Not only does clinically overt CVD increase with advancing age, but also subclinical or occult disease such as silent atherosclerosis. Therefore, is aging synonymous with cardiovascular 'disease'? This is not yet clear as many older people achieve old age without developing detectable CVD. Although at a population level age appears to be a significant risk factor for CVD, at an individual level there is variation among people with similar age and risk factor profiles. Age may be interpreted as increased exposure time to traditional CVD risk factors such as hypertension, diabetes, smoking, dyslipidemia, and sedentary lifestyle. Cardiovascular structure and function change with age and this may potentiate the effects of traditional risk factors in older people. Thus the increase in CVD seen in older people can be viewed as an interaction between age (exposure time), cardiovascular risk factors, and genetic predisposition [4]. The increased prevalence of vascular disease with advancing age is associated with a parallel increased prevalence of chronic kidney disease (CKD), partly exaggerated by the current definition and classification of CKD [5]. We suggest that the latter is more likely to be a manifestation of the former rather than vice versa. In this review, we summarize the vascular and renal changes associated with aging and explore whether they result from a natural physiological aging process or are the consequence of diffuse vascular pathology.

\section{Age-Related Changes}

\section{Vascular Changes}

The arterial system consists of a network of elastic large arteries and high resistance medium and small arteries and arterioles. Large arteries, by virtue of their distensibility, convert the pulsatile output of the heart into a continuous pattern of peripheral blood flow. Small arteries and arterioles are the main determinants of peripheral vascular resistance. With increasing age, the arterial intima becomes less smooth and displays endothelial dysfunction. In the media, there is proliferation of smooth muscle cells, a reduction of elastin content, and progressive adventitial and medial fibrosis. These changes lead to increased intima-media thickness (IMT), vascular wall calcifications as well as stiffness leading to loss of compliance of the aorta and major arteries [6]. Increased intima thickness may represent an early stage of atherosclerosis and could be a predictor of future cardiovascular events. However, age-dependent intima thickening has been demonstrated in humans in the absence of athero- sclerosis; thus excessive intima thickening does not invariably represent early atherosclerosis but may just reflect arterial aging [7]. On the other hand, increased stiffness and loss of compliance of major arteries leads to increased systolic arterial pressure, decreased diastolic pressure, and wide pulse pressure. This plays a central pathophysiological role in systolic hypertension in older people. Isolated systolic hypertension and a wide pulse pressure are determinants of cardiovascular risk [8]. As with intima thickening, vascular stiffening can occur independent of atherosclerosis [9]. Therefore, a combination of age-related vascular changes (intima and media thickening, wall stiffness, lumen dilatation, and decreased compliance), occurring to varying degrees, determine the overall vascular aging of the individual. Mild combinations could represent successful or healthy vascular aging, whilst severe and progressive combinations, associated with exposure to traditional risk factors, could lead to unsuccessful or pathological aging. The exact dividing line between normal and pathological aging is thus blurred. Atherosclerosis could be viewed not as a specific disease but as an interaction between age-related combinations of vascular changes and atherosclerotic risk factors [10]. In other words, worse combinations of age-related vascular changes provide the suitable substrate for the traditional cardiovascular risk factors to promote CVD. The individual variations in the occurrence of these worse combinations of age-related vascular changes could be genetically programmed and/or determined during fetal life or influenced by adverse growth patterns in early postnatal life [11].

\section{Renal Changes}

Aging is associated with renal structural changes and functional decline. It is not yet very clear how much of the functional loss is due to age per se and how much is related to associated CVD and life course exposure to vascular risk factors such as hypertension, diabetes and smoking. The major histological features of renal aging are arterial intimal fibrosis as well as glomerulosclerosis, tubular atrophy and interstitial fibrosis [12]. Earlier studies have suggested loss of renal mass, especially renal cortex, with advancing age. There is a $32 \%$ reduction in renal weight at autopsy of subjects over 80 years of age compared to those less than 39 years old [13]. In the renal cortex, the incidence of glomerulosclerosis increases from less than $5 \%$ at the age of 40 years to up to $30 \%$ by the 8 th decade [14]. This is associated with a simultaneous increase in vascular thickness; arterial and arteriolar hyalinosis and sclerosis. In the medulla, glomerulosclerosis 
is accompanied by the formation of a direct channel between afferent and efferent arterioles maintaining the medullary blood flow and potentially bypassing the glomerulus, leading to ischemia and reduction in function [15]. Thus, a combination of functional glomerulopenia and structural glomerulosclerosis leads to progressive reduction in glomerular filtration rate (GFR) with age [16]. However, these earlier studies should be interpreted with caution as they have assessed the effect of aging on renal function and structure by utilizing crosssectional studies in institutionalized elderly subjects. Older people living in care homes frequently suffer from comorbid conditions such as hypertension, diabetes and CVD that may have an additive effect on renal structure and function. This may confound and amplify the effect of age per se on renal structure and function.

However, as with CVD, not all the elderly display a decline in renal function with age. The Baltimore Longitudinal Study on Aging [17] reported an average decline in creatinine clearance of $0.75 \mathrm{ml} / \mathrm{min} /$ year after the $3 \mathrm{rd}$ decade of life, with approximately one third of subjects demonstrating stable kidney function over 20 years of follow-up. However, some have questioned the validity of such observations as the number of serial and repeated serum creatinine measurements were insufficient in many individuals to be able to ascertain the course of the renal function [18]. Another study showed a decline in GFR by $1.05 \mathrm{ml} / \mathrm{min} /$ year in the very elderly aged 70-110 years old [19]. Results from the Cardiovascular Health Study also indicate little or no progression of CKD in the majority of older people $(>26.5 \mu \mathrm{mol} / \mathrm{l}$ increase in serum creatinine in less than $3 \%$ of the subjects, mean age 73 years) over 3 years [20]. Fliser et al. [21] observed that GFR (although lower in older as compared to younger people) remained within the normal range in older normotensive subjects on normal dietary protein intake, and concluded that age per se is not the major determinant of the decline in GFR with age.

\section{Associations between Age-Related Renal Outcomes and CVD}

Earlier autopsy studies showed a positive association between the severity of atherosclerosis within renal arteries and the extent of glomerulosclerosis [22].

A recent prospective study of 4,380 community-based subjects (age $\geq 65$ years) has demonstrated the independent association of clinical atherosclerosis, heart failure, and subclinical measures of CVD with kidney function decline with aging, suggesting that kidney functional decline in the elderly is a result of microvascular atherosclerosis [23]. Among persons without clinical CVD, measures of subclinical atherosclerosis such as decreased ankle arm index, increased common carotid and internal carotid IMT were independently associated with rapid kidney function decline [23]. Also, the Cardiovascular Health Study showed that retinal microvascular abnormalities were associated with deterioration of renal function independent of the presence of diabetes or hypertension [24]. Increased carotid IMT was also associated with increased risk of incident CKD in a European prospective study of 2,751 subjects with normal renal function (GFR $>60 \mathrm{ml} / \mathrm{min} / 1.73 \mathrm{~m}^{2}$ ) at baseline followed up for 2 years [25]. However, in a recently published cross-sectional study IMT was not associated with a decline in renal function in a healthy Chinese population free of clinical CVD and with normal renal function (GFR $>60 \mathrm{ml} /$ $\min / 1.73 \mathrm{~m}^{2}$ ) after adjustment for cardiovascular risk factors [26]. A number of studies suggested that age-related loss of renal function is more pronounced in older people in whom CVD and risk factors coexist $[27,28]$. In addition, the associated comorbid conditions and ensuing vascular disease may also determine mortality in elderly individuals with CKD. In a large study of 27,998 individuals with GFR $<90 \mathrm{ml} / \mathrm{min} / 1.73 \mathrm{~m}^{2}$ followed up for 5 years, the incidence of end-stage renal disease (ESRD) was very low whilst mortality rate was much higher [29]. In a Norwegian prospective study, 3,069 of 65,604 people had CKD and the risk of ESRD was also especially low in people without diabetes or hypertension, women, and those aged $\geq 70$ years. In contrast, there was a high cardiovascular mortality among people with GFR $<45 \mathrm{ml} /$ $\mathrm{min} / 1.73 \mathrm{~m}^{2}$ [30]. This was confirmed in the large cohort of the Kaiser Permanente study in Northern California [31] where the sharpest increase in CVD risk was noted in albuminuric subjects with GFR $<45 \mathrm{ml} / \mathrm{min} / 1.73 \mathrm{~m}^{2}$. O'Hare et al. [32] investigated the age-specific incidence of death, treated ESRD, and change in GFR among 209,622 US veterans with CKD stages 3-5 followed up for a mean of 3.2 years. Among patients of all ages, rates of both death and ESRD were inversely related to GFR at baseline. However, among those with comparable levels of GFR, older patients had higher rates of death and lower rates of ESRD than younger patients. The level of GFR below which the risk of ESRD exceeded the risk of death varied by age, ranging from $45 \mathrm{ml} / \mathrm{min} / 1.73 \mathrm{~m}^{2}$ for 18 to 44-year-old patients to $15 \mathrm{ml} / \mathrm{min} / 1.73 \mathrm{~m}^{2}$ for 65 to 84 -year-old patients. Among those 85 years or older, the risk of death always exceeded the risk of ESRD. Previous 
studies have demonstrated that CKD is associated with an increased prevalence of traditional and non-traditional cardiovascular risk factors (elevated fibrinogen and Creactive protein as well as albuminuria) which may indicate that low-grade inflammation is responsible for both age-related vascular disease and decline in renal function [33]. The association of age-related vascular disease and microinflammation may also underlie the observed link between CVD and albuminuria. Microalbuminuria has consistently been shown to be associated with increased cardiovascular mortality. This was confirmed in patients with CKD stage 3 where the absence of albuminuria led to a good prognosis, unlike that of those with stages 1 and 2 with albuminuria [34]. A recent study also confirmed that the severity of albuminuria was proportional to the mortality of patients with congestive heart failure [35]. However, it is important to note that microalbuminuria is likely to reflect diffuse endothelial dysfunction and underlying vascular pathology involving renal glomerular capillaries.

\section{A Unifying Concept}

Aging is not a disease. It remains somewhat uncertain whether the age-related decline in renal function is associated with biological vascular aging or is due to chronological age per se. It is likely that the cumulative exposure to cardiovascular and consequently renal risk factors in certain genetically predisposed individuals results in what seems to be an age-related decline in renal function. It is likely that a worse combination of age-related vascular changes provide the suitable substrate for the traditional cardiovascular risk factors to promote CVD and the ensuing CKD.

A multi-hit hypothesis can therefore be put forward whereby genetically predisposed individuals or those whose risk emerges during the fetal or postnatal period develop CVD when exposed through a prolonged life course to cardiovascular risk factors such as hypertension, diabetes, smoking and/or dyslipidemia. CVD with the associated renal arterial, arteriolar and capillary (glomerular) damage leads to renal ischemia and progressive decline in kidney function. On such a compromised functional background, the rate of decline in kidney function in such individuals may be accelerated by further hits later in life such as episodes of acute kidney injury [36], drug nephrotoxicity or urinary tract obstruction. Further decline may also be associated with poor control of hypertension or diabetes. The observed varia- tions in kidney function in older people may therefore result from a combination of the above-described influences. Those with normal renal function would have a genetic background that did not predispose them to agerelated biological vascular aging and/or had minimal exposure to cardiovascular risk factors. There is no independent relationship between age-related decline in kidney function and atherosclerosis in a population with normal kidney function [26]. Those with impaired (GFR $<60 \mathrm{ml} / \mathrm{min} / 1.73 \mathrm{~m}^{2}$ ) but stable kidney function are likely to have suffered the cumulative effect of life course exposure to cardiovascular risk factors with ensuing ischemia and renal dysfunction. In the absence of additional intrinsic renal disease, proteinuria or ongoing kidney damage, they seldom progress to ESRD. By contrast, the elderly with progressive CKD and associated overt proteinuria either have a more severe and progressive form of vascular-renal pathology, with marked atherosclerosis/ glomerulosclerosis, or a progressive intrinsic renal disease such as chronic glomerulonephritis. Those are likely to progress to ESRD when exposed to superimposed, intercurrent, renal insults such as acute kidney injury, drug nephrotoxicity, obstructive uropathy or accelerated hypertension.

In conclusion, impaired kidney function in the elderly may represent a spectrum of physiological and pathological changes. Often, it consists of a combination of both, with those most severely affected representing a predominance of severe underlying vascular damage affecting a number of end organs including the kidneys. The presence of microalbuminuria is often a manifestation of systemic vascular damage. On the other hand, overt proteinuria reflects severe and progressive kidney disease.

This review highlights the links between CKD in the elderly and life course exposure to vascular damage and associated risk factors. It implies that the prevention of kidney impairment in the elderly should rely on the minimization of life-long exposure to cardiovascular risk factors.

\section{Acknowledgement}

We would like to thank Dr. Richard Glassock for his input and most constructive comments on the manuscript. 


\section{References}

$\checkmark 1$ Engberg H, Oksuzyan A, Jeune B, et al: Centenarians - a useful model for healthy aging? A 29-year follow-up of hospitalizations among 40,000 Danes born in 1905. Aging Cell 2009;8:270-276.

\2 Bulpitt CJ, Shipley MJ, Broughton PMG, et al: The assessment of biological age: a report from the Department of Environment Study. Aging (Milano) 1994;6:181-191.

$\checkmark 3$ Grundy SM: Age as a risk factor: you are as old as your arteries. Am J Cardiol 1999;83: 1455-1457.

$\checkmark 4$ Samani NJ, Harst P: Biological ageing and cardiovascular disease. Heart 2008;94:537539.

5 Winearls CG, Glassock RJ: Dissecting and refining the staging of chronic kidney disease. Kidney Int 2009;75:1009-1014.

-6 Franklin SS, Gustin W 4th, Wong ND, et al: Hemodynamic patterns of age-related changes in blood pressure. The Framingham Heart Study. Circulation 1997;96:308-315.

7 Virmani R, Avolio AP, Mergner WJ, et al: Effect of aging on aortic morphology in populations with high and low prevalence of hypertension and atherosclerosis: comparison between occidental and Chinese communities. Am J Pathol 1991;139:1119-1129.

$\checkmark 8$ Blacher J, Asmar R, Djane S, et al: Aortic pulse wave velocity as a marker of cardiovascular risk in hypertensive patients. Hypertension 1999;33:1111-1117.

$>9$ Avolio A: Genetic and environmental factors in the function and structure of the arterial wall. Hypertension 1995;26:34-37.

-10 Orlandi A, Marcellini M, Spagnoli LG: Aging influences development and progression of early aortic atherosclerotic lesions in cholesterol-fed rabbits. Arterioscler Thromb Vasc Biol 2000;20:1123-1136.

$\checkmark 11$ Nilsson PM, Lurbe E, Laurent S: The early life origins of vascular aging and cardiovascular risk: the EVA syndrome. J Hypertens 2008;26:1049-1057.

12 Zhou X J, Rakheja D, Yu X, et al: The aging kidney. Kidney Int 2008;74:710-720.

$>13$ Tauchi H, Tsuboi K, Okutomi J: Age changes in the human kidney of the different races. Gerontologia 1971;17:87-97.
14 Kaplan C, Pasternack B, Shah H: Age related incidence of sclerotic glomeruli in human kidneys. Am J Pathol 1975;80:227-234.

15 Takazakura E, Sawabu N, Handa A, et al: Intrarenal vascular changes with age and disease. Kidney Int 1972;2:224-230.

16 Hoang K, Tan JC, Derby G, et al: Determinants of glomerular hypofiltration in aging humans. Kidney Int 2003;64:1417-1424.

17 Lindeman RD, Tobin JD, Shock NW: Longitudinal studies on the rate of decline in renal function with age. J Am Geriatr Soc 1985;33: 278-285.

18 Glassock RJ, Winearls C: aging and the glomerular filtration rate: truths and consequences. Trans Am Clin Climatol Assoc 2009; 120:419-428.

19 Fehrman-Ekholm I, Skeppholm L: Renal function in the elderly ( $>70$ years old) measured by means of iohexol clearance, serum creatinine, serum urea and estimated clearance. Scand J Urol Nephrol 2004;38:73-77.

20 Bleyer AJ, Shemanski LR, Burke GL, et al: Tobacco, hypertension, and vascular disease: risk factors for renal functional decline in an older population. Kidney Int 2000;57:20722079.

-21 Fliser D, Zeier M, Nowack R, et al: Renal functional reserve in healthy elderly subjects. J Am Soc Nephrol 1993;3:1371-1377.

-22 Kasiske BL: Relationship between vascular disease and age associated changes in the human kidney. Kidney Int 1987;31:1153-1159.

23 Shlipak MG, Katz R, Kestenbaum B, et al: Clinical and subclinical cardiovascular disease and kidney function decline in the elderly. Atherosclerosis 2009;204:298-303.

24 Edwards MS, Wilson DB, Craven TE, et al: Association between retinal microvascular abnormalities and declining renal function in the elderly population: the Cardiovascular Health Study. Am J Kidney Dis 2005;46:214224.

25 Chonchol M, Gnahn H, Sander D: Impact of subclinical carotid atherosclerosis on incident chronic kidney disease in the elderly. Nephrol Dial Transplant 2008;23:25932598.

26 Han L, Bai X, Lin H, et al: Lack of independent relationship between age-related kidney function decline and carotid intimamedia thickness in a healthy Chinese population. Nephrol Dial Transplant 2010 [Epub ahead of print].
27 Baggio B, Budakovic A, Perissinotto E, et al: Atherosclerotic risk factors and renal function in the elderly: the role of hyperfibrinogenaemia and smoking. Results from the Italian Longitudinal Study on aging (ILSA). Nephrol Dial Transplant 2005;20:114-123.

28 de Lusignan S, Chan T, Gallagher H, et al: Chronic kidney disease management in southeast England: a preliminary cross-sectional report from the QICKD - Quality Improvement in Chronic Kidney Disease study. Prim Care Cardiovasc J 2009;2:33-39.

$\checkmark 29$ Keith DS, Nichols GA, Gullion CM, et al: Longitudinal follow-up and outcomes among a population with chronic kidney disease in a large managed care organization. Arch Intern Med 2004;164:659-663.

30 Hallan SI, Dahl K, Oien CM, et al: Screening strategies for chronic kidney disease in the general population: follow-up of cross sectional health survey BMJ 2006;333:1047.

31 Go AS, Chertow GM, Fan D, et al: Chronic kidney disease and the risks of death, cardiovascular events, and hospitalization. N Engl J Med 2004;351:1296-1305.

32 O'Hare AM, Choi AI, Bertenthal D, et al: Age affects outcomes in chronic kidney disease. J Am Soc Nephrol 2007;18:2758-2765.

33 Muntner P, Hamm L, Kusek JW, et al: The prevalence of non traditional risk factors for coronary heart disease in patients with chronic kidney disease. Ann Intern Med 2004;140:9-17.

34 Brantsma AH, Bakker SJ, Hillege HL, et al; PREVEND Study Group: Cardiovascular and renal outcome in subjects with K/DOQI stage 1-3 chronic kidney disease: the importance of urinary albumin excretion. Nephrol Dial Transplant 2008;23:3851-3858.

$\checkmark 35$ Jackson CE, Solomon SD, Gerstein HC, et al; CHARM Investigators and Committees: Albuminuria in chronic heart failure: prevalence and prognostic importance. Lancet 2009;374:543-550.

36 Ishani A, Xue JL, Himmelfarb J, et al: Acute kidney injury increases risk of ESRD among elderly. J Am Soc Nephrol 2009;20:223-228. 


\section{Editorial Comment}

R.J. Glassock, Laguna Niguel, Calif.

The mini-review by Abdelhafiz and colleagues from Rotherham General Hospital and the Sheffield Kidney Institute in the United Kingdom addresses a controversial area of clinical nephrology; namely, is the decline in kidney function seen in aging a consequence of normal physiological processes (renal senescence) or a pathological process (nephrosclerosis) or some admixture of both? They conclude that only a fraction of elderly subjects with a decline in kidney function actually have underlying progressive vascular pathology, amenable to modification by specific interventions. It is a given that aging is inevitable, but is chronic kidney disease (CKD; as presently defined generically by the threshold of estimated GFR uncorrected for the effects of aging) a dangerous and treatable condition in many elderly subjects? The authors forcibly and logically argue that aging should not be regarded as a disease per se but that the physiological changes in the kidneys and the vasculature in general serve as a nutritive substrate for the co-development of what we call cardiovascular disease. This mixture may predispose to episodes of acute kidney injury or even the development of progressive CKD and end-stage renal disease when poor control of hypertension, diabetes mellitus or dyslipidemia supervenes. They stress, importantly in my opinion, that excretion of small quantities of albumin in the urine (microalbuminuria) is most properly viewed as a form of systemic vascular damage rather than a kidney disease in its own right. In their construct, progressive CKD in the elderly is seen as a consequence of systemic vascular disease superimposed on normal physiological aging of the kidneys rather than a primary kidney disease as the root cause of the vascular disease in the elderly. This emphasizes the true nature of the 'cardio-renal syndrome' which some have also erroneously called the reno-cardiac syndrome. We need to put the cart before the horse in our understanding of the connections between aging, vascular and kidney disease in the elderly. The concepts enunciated in this timely minireview have significant implications for the definition of CKD as well as for the design of interventional strategies used for impacting the burden of vascular and bona-fide kidney disease in the elderly. 